Minireview

\title{
The future of artemisinins: natural, synthetic or recombinant? Marcel Hommel
}

Address: Institut Pasteur, 25-28 rue du Dr Roux, Paris 75724 Cedex 15, France. Email: mhommel@pasteur.fr

Published: 15 December 2008

Journal of Biology 2008, 7:38 (doi:10.1 I86/jbioll 0I)

The electronic version of this article is the complete one and can be found online at http://jbiol.com/content/7/10/38

(c) 2008 BioMed Central Ltd

\begin{abstract}
Artemisinins are the most important anti-malarial drugs in use today, but are more costly than previous anti-malarials and production and price tend to fluctuate. Alternative ways of producing artemisinins are discussed here in the light of a recent paper in BMC Biotechnology on improving the yield of the precursor, artemisinic acid, in genetically engineered yeast.
\end{abstract}

Artemisinin, derived from sweet wormwood, Artemisia annua, is the spearhead of anti-malarial chemotherapy. In 2004, the World Health Organization (WHO) recommendation that artemisinin-based combination therapy (ACT) should be the norm for the treatment of falciparum malaria in most endemic countries came into effect [1]. Financial backing for this treatment from the Global Fund for AIDS, Tuberculosis and Malaria and other international sponsors has led to a massive increase in the demand for artemisinin, but with an estimated 12-14 months lead-time from planting of $A$. annua to artemisinin extraction, stepping-up production (or adjusting to fluctuations in demand) from the plant is not simple, and alternative means of producing the raw material by complete chemical synthesis or recombinant technologies are being explored. It would, however, be naive to believe that the cost of the raw material is the main determinant of the cost of a drug. This article looks at issues surrounding the production of artemisinins, particularly in the light of a recent paper by Jay Keasling and colleagues (Ro et al. [2]) in BMC Biotechnology, which describes a way of improving the yield of the precursor, artemisinic acid, in genetically engineered yeast.

\section{The chemistry and therapeutic applications of artemisinins}

Artemisinin (qinghaosu) was discovered in 1971 by Chinese scientists looking at the anti-malarial properties of herbs in the traditional Chinese pharmacopeia: A. annua had been used as an antipyretic tea for more than 2,000 years. Although no longer used as a drug itself, artemisinin is the starting compound for a class of highly active anti-malarials, which are produced by chemical modification of artemisinin. These 'artemisinins' include dihydroartemisinin, artemether and artesunate, the latter being a particularly interesting derivative because it is water-soluble and can be given intravenously.

Artemisinin is a sesquiterpene lactone containing an endoperoxide bridge that is considered essential to its antimalarial action: haem-catalyzed cleavage of the peroxide generates unstable free radicals to which malaria parasites are particularly sensitive [3]. Although the peroxide activity is necessary, it may not be sufficient to explain the mechanism of action of artemisin, and alternative hypotheses might need to be considered. Specific interactions of artemisinin with parasite proteins, such as the translationally controlled tumor protein (TCTP) and the parasite ortholog of sarcoplasmic/endoplasmic-reticulum $\mathrm{Ca}^{2+}$-ATPase (PfATP6), have been reported, but the interaction with haem within the parasite-containing vacuole inside the host cell is of special interest. The conversion of toxic haem into non-toxic hemozoin, or malarial pigment, is crucial for parasite survival, and the inhibition of hemozoin formation is a major mechanism of action of many existing anti-malarials, 


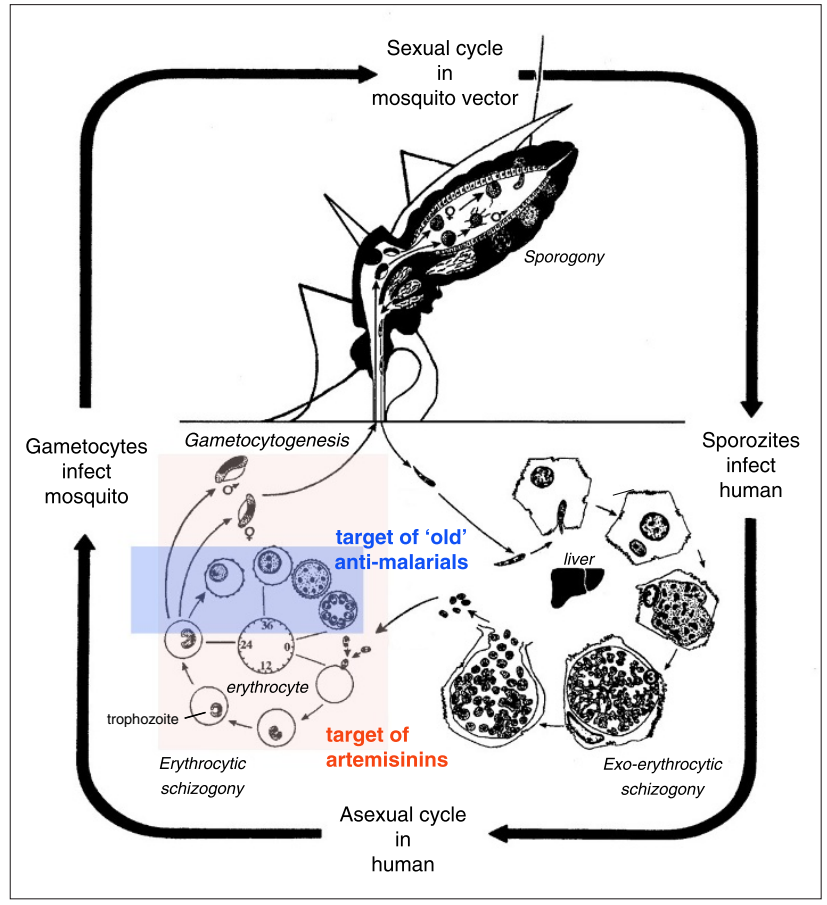

Figure I

Artemisinins kill parasites more effectively and at an earlier stage in the erythrocytic part of its life cycle than most of the other anti-malarial currently in use. They also kill the gametocyte stage and may contribute to interrupting transmission. They do not work on the exo-erythrocytic forms, hence do not prevent relapses in $P$. vivax or $P$. ovale.

including chloroquine. Kannan et al. [4] have shown that there is a potent interaction between protein-bound haem in hemoglobin and artemisinin, with the formation of 'hemarts', which bind to PfHRP2 (Plasmodium falciparum histidine-rich protein-2) and inhibit hemozoin formation.

Artemisinins have broad stage specificity in their antimalarial action, killing all asexual stages, as well as gametocytes, but have no effect on the exo-erythrocytic stages. Where artemisinins differ from other anti-malarials is that they kill young trophozoites ('ring' stage); (Figure 1). As a result, artemisins are rapidly effective and reduce the number of late trophozoites in the capillaries. There is a rapid clinical improvement in patients and reduction of the risk of severe complications. The effect on gametocytes means less efficient transmission of the parasite to the mosquito vector, which potentially contributes to an interruption of transmission to humans and also reduces the risk of dispersion of any drug-resistant mutants. Recent field studies have shown that artemisinin treatment significantly reduced gametocyte carriage compared with other drugs, an effect that was variable from one geographical area to another, depending on the level of malaria endemicity and access to treatment [5].
Monotherapy with artemisinins has now mostly been abandoned. WHO finally agreed, after years of lobbying, to recommend the use of a three-day regimen of combination therapy - that is, artemisinin together with a partner drug for the treatment of uncomplicated malaria. Because of their different kinetics of action, not only does one drug protect the other from provoking resistance, but as artemisinins effectively kill most of the parasites in the first few hours of treatment, the partner drug has a better chance of being effective against the remaining few [6]. Two fixed-dose drug combinations are available, artemether-lumefantrine (Coartem or Riamet) and artesunate-amodiaquine (Coarsucam or ASAQ): these have broadly comparable efficacy and the fixed dose has many advantages over other presentations, such as blister-packs. Intravenous artesunate acts significatively faster than intravenous quinine, in terms of both parasite clearance and clinical improvement, and there is now a strong case for recommending parenteral artesunate instead of quinine for the treatment for severe malaria [7].

As well as their anti-malarial activity, artemisinins are effective against a number of other parasitic diseases, particularly schistosomiasis, and there is now growing experimental evidence that they may potentially be useful against cancer, especially as their mechanism of action is so different from that of other anti-cancer drugs [8].

\section{How much artemisinin is needed?}

This question can be rephrased as how many cases of malaria need to be treated with ACT every year?' and is difficult to answer. Estimates of the true prevalence of malaria worldwide vary greatly because they are not based on accurate reporting at the country level. In most endemic countries, treatment is given on a presumptive diagnosis, sometimes using a clinical algorithm with the presence of fever as a key factor but generally without laboratory confirmation. At best this approach is only $50 \%$ reliable (and less than $5 \%$ reliable in areas of low endemicity). In Africa alone, various estimates suggest that there are 215 to 375 million malaria episodes per year, and, on the basis of usage of the 'old anti-malarials', Kindermans et al. [9] calculated projected drug consumption to be between 113 and 314 million adult-equivalent treatment courses per year. In 2004, the forecast was for 60 million treatment courses in 2005 and 130 million in 2006. Although these figures already underestimated actual medical need, the amount of ACT delivered in Africa in 2006 was even lower (less than 100 million treatment courses). Forecasting artemisinin needs is clearly a highly complex process. Underproduction leads to shortages and inflated prices, whereas overproduction leads to rapid expiry of the drugs and potential losses by suppliers, as outlined by Cohen and colleagues [10], who looked at forecasting issues from a funding agency angle. 


\section{Natural artemisinins}

A. annua is an annual plant, growing to $150 \mathrm{~cm}$ tall and found wild in many countries (Figure 2). Production of $A$. annua is dominated by China and Vietnam, but it is also now grown in the highlands of Tanzania, South Africa, India and Madagascar. The active pharmaceutical ingredient (API) or 'raw artemisinin' is extracted from the leaves, just before the plant flowers, and from planting to extraction takes more than 12 months. Soil, climate, altitude and grower's know-how can affect the content of artemisinin substantially. Early problems with scaling-up production have, to a large extent, been solved by selection of plants for high artemisinin content and adaptation to the environment in which they are to be grown. Further improvement in yield and quality may be possible by further selection, like that being undertaken by Artemisinin Enterprise at the University of York, in collaboration with the Swiss not-forprofit organization Médiplant, using fast-track genetic breeding technologies. Hydroponics and in vitro plant culture are other interesting options, which have not yet confirmed their potential.

There are claims that the plant simply infused in water could provide a 'home-grown' malaria treatment in rural areas in the tropics. Although trials of such a tea yielded higher than expected amounts of artemisinin, and the plasma concentration achieved by consumption of the tea was compatible with an effective anti-malarial activity (reviewed in [11]), the same trials showed considerable variation in artemisinin content from one batch of plants to another and a high rate of malaria recrudescence. It would be wrong to promote the use of wormwood tea as a 'cheap' alternative to drug treatment. This use would certainly increase the risk of resistance emerging and A. anпиа does not grow in the tropical environment where malaria is endemic and cannot, therefore, be 'home-grown' where it would be most needed.

Raw artemisinin is extracted with solvents, such as hexane, followed by chromatography to improve purity. The extracts usually contain both artemisinin and its precursor, artemisinic acid, which can later be transformed into artemisinin. On average, 40 hectares (ha) of land produce a tonne of dried leaves, which can yield $6 \mathrm{~kg}$ of artemisinin. The production of 100 million adult treatment courses would require about 10,000 ha of $A$. апnиa plantations. Despite the relatively low yield, the exploitation of $A$. annua is commercially viable as long as the price of API is sufficiently stable. In reality, there have been massive fluctuations since 2004, with prices varying between US $\$ 1,600 / \mathrm{kg}$, when there was a world shortage following the WHO recommendation, to as low as $\$ 150$ at the end of 2007, when there was a glut. This see-saw effect, which is a direct

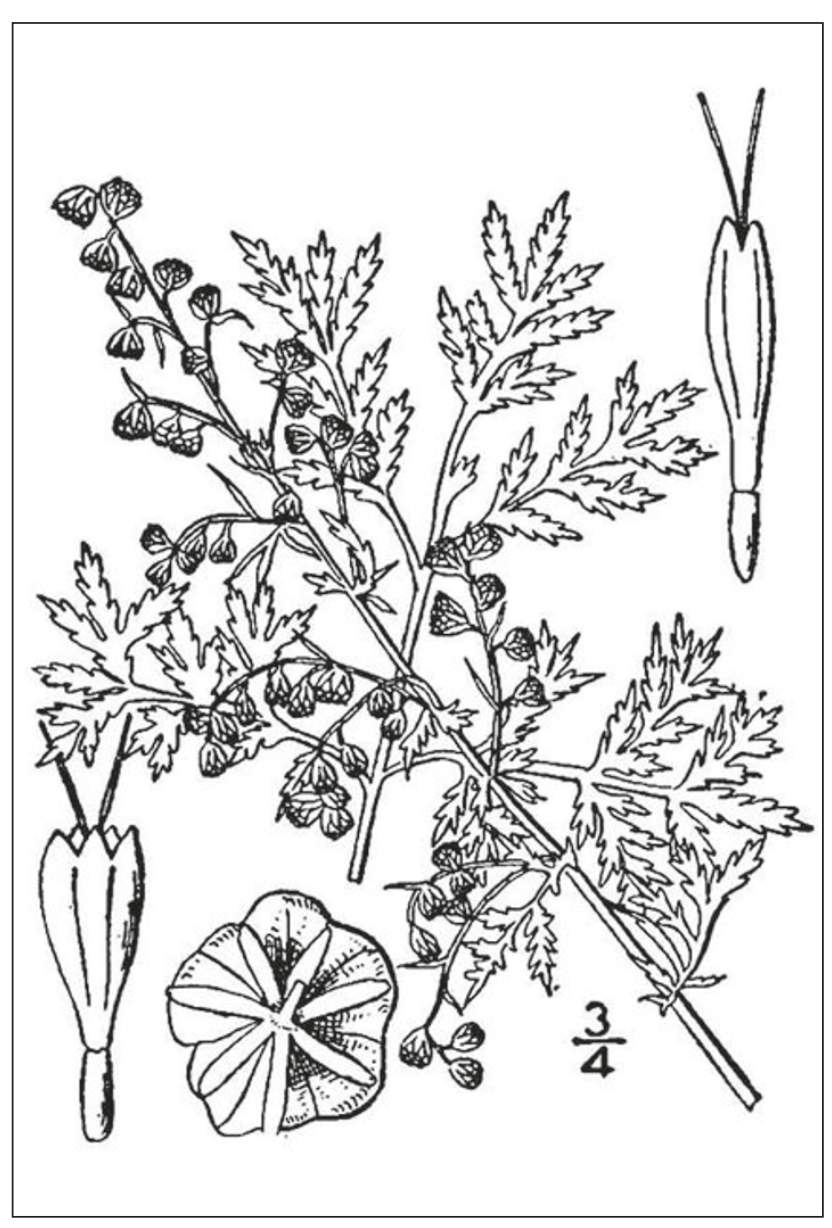

Figure 2

Artemisia annua plant. USDA-NRCS PLANTS Database, NL Britton and A Brown. 1913. Illustrated flora of the northern states and Canada. Vol. 3: 526 .

result of the problems in forecasting demand, is not sustainable in the long term and efforts are being made to stabilize the market [12].

Even more than cost, the difficulty in coping with fluctuations in the supply of raw artemisinin of plant origin is the major pressure for a switch to alternative sources. It is considerably easier to cope with ups and downs in demand by switching on a chemical synthesis or a fermentation process than by dealing with the long lead-time of plantations.

The drugs currently used as components of ACT are no longer 'natural' artemisinins, as extraction from the plant is followed by a semi-synthetic process to transform the molecule first into dihydroartemisinin, then into artemether or artesunate. Another alternative, which is, surprisingly, not used industrially outside China, is to focus the extraction 
process entirely on artemisinic acid, which being 8-10 times more abundant in the plant gives much better yields, and then to use one of the conversion processes described to transform it into artemisinin.

\section{Alternatives to production from plants}

The full chemical synthesis of artemisinin was achieved in 1983, but is too complex and expensive for commercialization. The alternative is the development of fully synthetic peroxides (trioxanes or trioxolanes). These molecules have similarities with artemisinin, including the key endoperoxide bridge, but their mode of action is different in many ways. Of the many peroxides tested, one trioxolane showed promise (OZ277/RBx11160 or Arterolane) [13]: it was highly potent against $P$. falciparum in vitro and its synthesis was sufficiently simple for industrial scale-up. The drug proved too unstable in vivo, however, and clinical trials were stopped before phase III. More stable molecules are in development and a hybrid trioxane-aminoquinoline (trioxaquine) [14] may achieve the looked-for objective of a fully synthetic anti-malarial peroxide.

Microbial genetic engineering is an attractive alternative to chemical synthesis. Artemisinic acid can be produced in Escherichia coli and, even more effectively, in Saccharomyces cerevisiae [15], following pioneering work by Keasling and colleagues, who transferred $10 \mathrm{~A}$. annua genes into E. coli to create an almost 'plant-like' environment in the bacteria. Their approach circumvents some of the previous problems of engineering the precursors (amorphadiene or artemisinic acid) or enzymes (amorphadiene oxidase) involved in the artemisinin biosynthetic pathway. Expression levels in yeast are still insufficient for industrialized scale-up, but the new publication by the group [2] describes a further improvement in production in yeast (with a 10 times higher yield) by using a high-copy plasmid system. This opens the way for a production process that would completely bypass the need for plantations of $A$. annua, and in which the yeastproduced precursor would be chemically converted to artemisinin as indicated above. There are still a few hurdles to clear, including confirmation of structural and functional identity between the natural and engineered molecules.

\section{The threat of drug resistance}

We know from past experience that, once emerged, resistance of the malaria parasite to a drug spreads fast: chloroquine resistance emerged in the late 1950s in SouthEast Asia, was reported for the first time in East Africa in the 1970s, and has since invaded the entire African continent. It is probably inevitable that mutants resistant to artemisinins will eventually emerge and, if they do, this will probably happen in South-East Asia, where artemisinins have been used extensively as monotherapy for nearly 20 years. Studies from the Thai-Cambodian border, and more recently from South Cambodia, have already shown reduced susceptibility, as well as treatment failure rates with ACT exceeding 10\%, which is higher than anywhere else. This area is now carefully observed by the scientific community, but monitoring is difficult in the absence of reliable molecular markers for artemisinin resistance, and relies on in vivo and in vitro sensitivity testing, which is cumbersome. As long as there is no resistance to the partner drug, it is usually assumed that the risk of resistance spreading will remain low. It is important that malaria treatment be most carefully controlled in South-East Asia, banning monotherapy (which is still frequent), ensuring access to prompt ACT treatment with good-quality drugs and, whenever possible, treating asymptomatic carriers, who contribute to the spread of resistance.

In conclusion, even when synthetic or recombinant artemisinin-like molecules have gone through preliminary tests to confirm their worth, there will still be a long way to go - at least five to six years and millions of dollars - before they will be available as drugs. It is, therefore, likely that $A$. annua will continue to be the main source of artemisinin for the foreseable future. It is true that the effectiveness of increasing worldwide utilization of ACT relies on a sustainable supply of artemisinin, preferably at an affordable price. It is also true that limited access to ACT, as is still the case in many African countries, is not necessarily due to a shortage of raw material, or insufficient production, or even the high cost of the drug. It is more often a poor coordination of distribution at country level. Major funding organizations, including the Global Fund, have begun to understand that, in order to be effective, they not only have to deal with the cost of the drug, but must also strengthen health-system infrastructure and improve provision for diagnosis and treatment in the field. Keeping the cost of the drug down means not only ensuring a sustainable supply of the raw material, but also putting pressure on manufacturers to keep the price of the final drug reasonable; both of which could be achieved by using subsidies to preorder drugs years in advance. This, however, assumes that forecasting need becomes much better than is currently feasible. The recent endorsement by the 2008 Roll Back Malaria Action Plan of the Affordable Medicines Facility for malaria (AMFm) initiative, a proposed $\$ 1.9$ billion financial resource, is likely to have a major role in improving access to the drug at all levels.

\section{References}

I. World Heath Organization: Guidelines for the Treatment of Malaria. WHO/HTM/MAL/2006. I I 08.

2. Ro DK, Ouellet M, Paradise EM, Burd H, Eng D, Paddon CJ, Newman JD, Keasling JD: Induction of multiple pleiotropic drug 
resistance genes in yeast engineered to produce an increased level of anti-malarial drug precursor, artemisinic acid. BMC Biotechnol 2008, 8:83.

3. Meshnick SR, Jefford CW, Posner GH, Avery MA, Peters W: Second-generation antimalarial endoperoxides. Parasitol Today 1996, 12:79-82.

4. Kannan R, Kumar K, Sahal D, Kukreti K, Chauhan VS: Reaction of artemisinin with haemoglobin: implications for antimalarial activity. Biochem J 2005, 385:409-4I8.

5. Stepniewska K, Price RN, Sutherland CJ, Drakeley CJ, von Seidlein L, Nosten F, White NJ: Plasmodium falciparum gametocyte dynamics in areas of different malaria endemicity. Malar J 2008, 7:249.

6. White NJ: Qinghaosu: the price of success. Science 2008, 320:330334.

7. Dondorp A, Nosten F, Stepniewska K, Day N, White N, South East Asian Quinine Artesunate Malaria Trial (SEAQUAMAT) group: Artesunate versus quinine for treatment of severe falciparum malaria: a randomised trial. Lancet 2005, 366:717-725.

8. Krishna S, Bustamante L, Haynes RK, Staines HM: Artemisinins: their growing importance in medicine. Trends Pharmacol Sci 2008, 29:520-527.

9. Kindermans JM, Vandenbergh D, Vreeke E, Olliaro P, D'Altilia JP: Estimating antimalarial drugs consumption in Africa before the switch to artemisinin-based combination therapies (ACTs). Malar J 2007, 6:91.

10. Cohen JM, Singh I, O'Brien ME: Predicting Global Fund grant disbursements for procurement of artemisinin-based combination therapies. Malar J 2008, 7:200.

II. De Ridder S, van der Kooy F, Verpoorte R: Artemisia annua as a self-reliant treatment for malaria in developing countries. J Ethnopharmacol 2008, 120:302-314.

12. Kindermans JM, Pilloy J, Olliaro P, Gomes M: Ensuring sustained ACT production and reliable artemisinin supply. Malar J 2007, 6:125.

13. Vennerstrom JL, Arbe-Barnes S, Brun R, Charman SA, Chiu FC, Chollet J, Dong Y, Dorn A, Hunziker D, Matile H, Mclntosh K, Padmanilayam M, Santo TJ, Scheurer C, Scorneaux B, Tang Y, Urwyler H, Wittlin S, Charman WN: Identification of an antimalarial synthetic trioxolane drug development candidate. Nature 2004, 430:900-904.

14. Benoit-Vical F, Lelièvre J, Berry A, Deymier C, Dechy-Cabaret O, Cazelles J, Loup C, Robert A, Magnaval JF, Meunier B: Trioxaquines are new antimalarial agents active on all erythrocytic forms, including gametocytes. Antimicrob Agents Chemother 2007, 5I: | 463-I472.

15. Ro DK, Paradise EM, Ouellet M, Fisher KJ, Newman KL, Ndungu JM, Ho KA, Eachus RA, Ham TS, Kirby J, Chang MC, Withers ST, Shiba Y, Sarpong R, Keasling JD: Production of the antimalarial drug precursor artemisinic acid in engineered yeast. Nature 2006, 440:940-943. 